\title{
CORRIGENDUM
}

\section{Hypertension is an important risk determinant for chronic kidney disease: results from a cross-sectional, observational study from a rural population in South India}

YJ Anupama, SN Hegde, G Uma and M Patil

Journal of Human Hypertension (2017) 31, 369-370; doi:10.1038/jhh.2017.4

Correction to: Journal of Human Hypertension (2017) 31, 327-332; doi:10.1038/jhh.2016.81; published online 24 November 2016

Since the online publication of this article the authors realised there were errors in Figure 1, Table 3 and Table 5.

In Figure 1, the value for 'non-availability at home during the time of sample collection' was given as $n=310$. This should be $n=410$. The corrected version of Figure 1 can be seen below.

In Table 3, the number of hypertensive males was given as 472 and the number of non-hypertensive males was given as 1003 . The correct number of hypertensive males is 202 and the correct number of non-hypertensive males is 383 . The corrected version of Table 3 can be seen below.

In Table 5, the symbol 'greater than' was inadvertently added to the odds ratio for HTN adjusted for age. The corrected version of Table 5 can be seen below.

The authors would like to apologise for any inconvenience caused by these errors.

These errors have now been rectified, and the corrected article appears in this issue. The html and online pdf versions have also been rectified, and now carry the corrected paper.

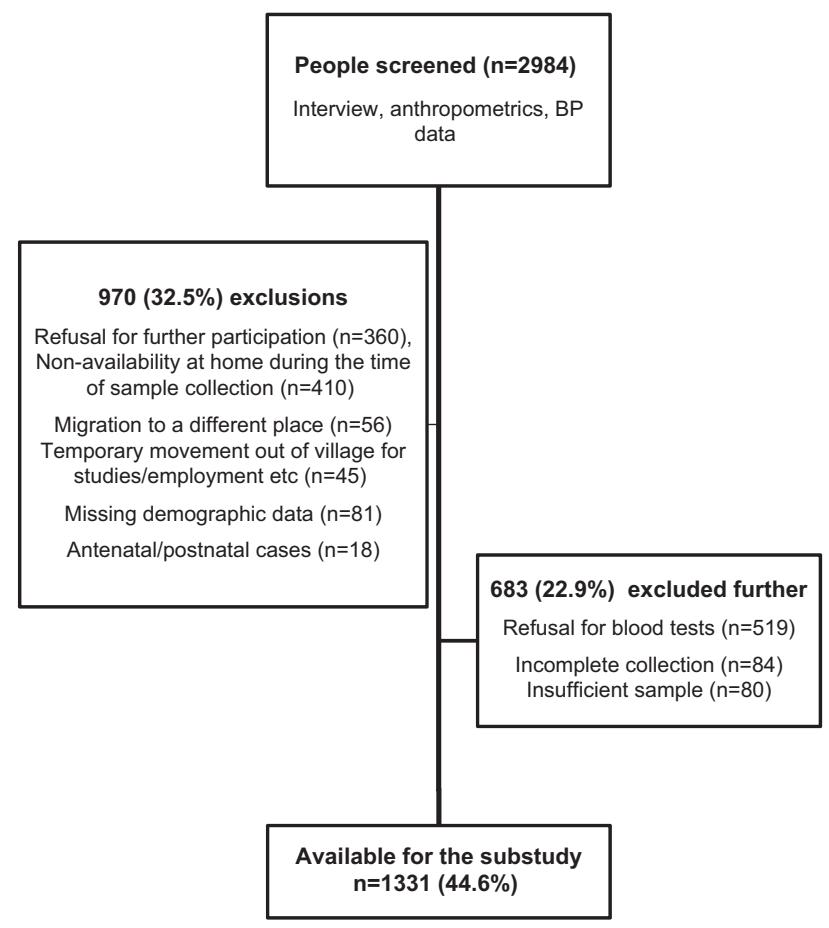

Figure 1 
Table 3. Determinants of hypertension in univariate analysis

\begin{tabular}{|c|c|c|c|c|}
\hline \multirow[t]{2}{*}{ Determinant } & \multirow{2}{*}{$\frac{\text { Hypertensives }(\mathrm{N}=434)}{\text { Number (\%) }}$} & \multirow{2}{*}{$\begin{array}{c}\text { Non-hypertensives }(\mathrm{N}=897) \\
\text { Number (\%) }\end{array}$} & \multicolumn{2}{|c|}{ Univariate } \\
\hline & & & P-value & OR $(95 \% \mathrm{Cl})$ \\
\hline Males & $202(46.5)$ & $383(42.6)$ & 0.185 & $0.86(0.68-1.07)$ \\
\hline Occupation (farming and labour) & $231(53.2)$ & $453(50.5)$ & 0.351 & $1.12(0.89-1.40)$ \\
\hline Smoking & $39(8.9)$ & $77(8.5)$ & 0.807 & $1.05(0.70-1.57)$ \\
\hline Alcohol & $42(9.6)$ & $68(7.5)$ & 0.193 & $1.31(0.87-1.95)$ \\
\hline Abdominal obesity & 375 (86.4) & $762(84.9)$ & 0.48 & $1.13(0.81-1.57)$ \\
\hline Diabetes & $53(12.2)$ & $35(3.9)$ & $<0.001$ & $3.43(2.20-5.34)$ \\
\hline Proteinuria & $21(4.8)$ & $16(1.8)$ & 0.001 & $2.8(1.45-5.42)$ \\
\hline Microalbuminuria & $23(5.3)$ & $25(2.7)$ & 0.021 & $1.95(1.10-3.48)$ \\
\hline eGFR $\left(<60 \mathrm{ml} \mathrm{min}^{-1} 1.73 \mathrm{~m}^{-2}\right)$ & $21(4.8)$ & $14(1.6)$ & $<0.001$ & $3.21(1.62-6.37)$ \\
\hline
\end{tabular}

Abbreviations: $\mathrm{BMI}$, body mass index; eGFR, estimated glomerular filtration rate; OR, odds ratio; $\mathrm{Cl}$, confidence intervals. Significant associations denoted by the bold-type font.

Table 5. Association of hypertension with chronic kidney disease

\begin{tabular}{llr}
\hline Variable & \multicolumn{2}{c}{ Chronic kidney disease } \\
\cline { 2 - 3 } & P-value & Odds ratio $(95 \% \mathrm{Cl})$ \\
\hline HTN & $<\mathbf{0 . 0 0 1}$ & $\mathbf{2 . 4 6}(\mathbf{1 . 6 4 - 3 . 6 9 )}$ \\
HTN adjusted for age & $<\mathbf{0 . 0 0 1}$ & $\mathbf{2 . 0 5}(\mathbf{1 . 3 3 - 3 . 1 8 )}$ \\
HTN adjusted for DM, BMI $>25$ & $<\mathbf{0 . 0 0 1}$ & $\mathbf{2 . 2 7}(\mathbf{1 . 5 0 - 3 . 4 3 )}$ \\
HTN adjusted for DM, BMI $>25$ & $<\mathbf{0 . 0 0 1}$ & $\mathbf{2 . 2 2}(\mathbf{1 . 4 6 - 3 . 3 6 )}$ \\
and habits & & \\
\hline Abbreviations: BMl, body mass index; Cl, confidence interval; DM, diabetes \\
mellitus; HTN, hypertension. Significant associations denoted by the bold- \\
type font.
\end{tabular}

